\title{
Allergic Bronchopulmonary Aspergillosis Presenting With Lung Mass-Report of Six Cases
}

\author{
Irfan $\mathbf{M}^{1}$, Jabeen $\mathrm{K}^{2}$, Iqbal $\mathbf{N}^{1}$, Farooqi JQ², Khan JA ${ }^{1}$
}

1 Section of Pulmonary and Critical care, Department of Medicine, Aga Khan University, Karachi, Pakistan 2 Department of Pathology and Laboratory Medicine, Aga Khan University, Karachi, Pakistan

\section{Background}

Allergic bronchopulmonary aspergillosis (ABPA) is a disorder caused by hypersensitivity to Aspergillus antigens. Patients with ABPA can have diverse radiological manifestations; however ABPA presenting as a lung masses is a rare presentation. So far, to our knowledge only 10 cases have been reported in literature. Here we report 6 patients of ABPA who presented with lung mass leading to lung cancer as a differential diagnosis.

Objective:

To study the clinical, radiological characteristics and outcome of patients of ABPA presented with a lung mass mimicking lung cancer.

\section{Methods:}

Medical records of 6 patients of ABPA presenting with lung mass at Aga Khan University hospital (AKUH), Karachi-Pakistan were reviewed. Diagnosis of ABPA in all cases was made using the Rosenberg criteria. Complete resolution of mass after steroid therapy was also used as an adjunct to diagnosis.

\section{Results:}

\begin{tabular}{|c|c|c|c|c|c|c|c|c|}
\hline Patient & Age / Sex & $\begin{array}{l}\text { History of } \\
\text { Asthma }\end{array}$ & Chest pain & Hemoptysis & $\begin{array}{l}\text { Location of } \\
\text { opacity }\end{array}$ & $\begin{array}{l}\text { Bronchoscopy } \\
\text { findings }\end{array}$ & Work up for ABPA & Clinical course \\
\hline 1 & $48 / M$ & Yes & tve & -ve & Lt. upper lobe & $\begin{array}{l}\text { LLL stenosis } \\
\text { and mucus } \\
\text { plugging }\end{array}$ & $\begin{array}{l}\text { IgE: }>\mathbf{2 0 0 0} \\
\text { TEC: } 2300 / \mu L \\
\text { AST:+ve }\end{array}$ & $\begin{array}{l}\text { Resolution } \\
\text { with } \\
\text { Prednisolone }\end{array}$ \\
\hline 2 & $32 / F$ & No & +ve & -ve & Rt. upper lobe & $\begin{array}{l}\text { CT guided } \\
\text { Biopsy (Non } \\
\text { specific) }\end{array}$ & $\begin{array}{l}\text { IgE: } 2326 \\
\text { TEC: } 1800 / \mu L \\
\text { AST::+ve }\end{array}$ & $\begin{array}{l}\text { Thoracotomy } \\
\text { \& resection }\end{array}$ \\
\hline 3 & $23 / M$ & Yes & +ve & tve & Lt. upper lobe & Not done & $\begin{array}{l}\text { IgE: } 2880 \\
\text { TEC: } 300 / \mu L \\
\text { AST:+ve }\end{array}$ & $\begin{array}{l}\text { Resolution } \\
\text { with } \\
\text { Prednisolone }\end{array}$ \\
\hline 4 & $30 / \mathrm{M}$ & Yes & tve & -ve & Rt. upper lobe & Not done & $\begin{array}{l}\text { IgE: }>3000 \\
\text { TEC: } 1300 / \mu \mathrm{L} \\
\text { AST:+ve }\end{array}$ & $\begin{array}{l}\text { Resolution } \\
\text { with } \\
\text { Prednisolone }\end{array}$ \\
\hline 5 & $50 / \mathrm{M}$ & Yes & tve & tve & Rt. upper lobe & $\begin{array}{l}\text { Mucus } \\
\text { plugging } \\
\text { right upper } \\
\text { lobe }\end{array}$ & 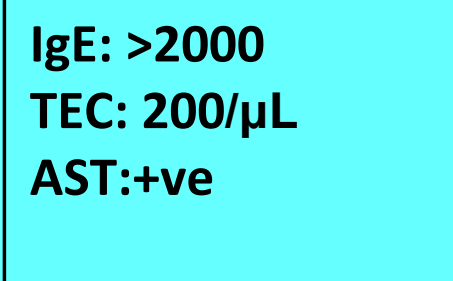 & $\begin{array}{l}\text { Resolution } \\
\text { with } \\
\text { Prednisolone }\end{array}$ \\
\hline 6 & $26 / M$ & No & +ve & tve & Rt. Upper lobe & Not done & $\begin{array}{l}\text { IgE: }>2000 \\
\text { TEC: } 800 / \mu \mathrm{L} \\
\text { AST:+ve }\end{array}$ & $\begin{array}{l}\text { Resolution } \\
\text { with } \\
\text { Prednisolone }\end{array}$ \\
\hline
\end{tabular}

AST: Aspergillus skin test; TEC: total eosinophil count/ $\mu \mathrm{L}$

\section{Conclusion:}

ABPA can present as pulmonary mass lesions and must be considered in the differential diagnosis of mass like lesions in lung, particularly in a patient with a history of asthma. Good clinical history, peripheral eosinophilia and raised $\lg \mathrm{E}$ levels can help in narrowing the differential diagnosis. Treatment with glucocorticoids is associated with excellent outcomes. 